\title{
Phase space description of nonlocal teleparallel gravity
}

\author{
Kazuharu Bamba $^{1, \mathrm{a}}$, Davood Momeni ${ }^{2, \mathrm{~b}}{ }_{\mathbb{D}}$, Mudhahir Al Ajmi $^{2, \mathrm{c}}$ \\ ${ }^{1}$ Division of Human Support System, Faculty of Symbiotic Systems Science, Fukushima University, Fukushima 960-1296, Japan \\ ${ }^{2}$ Department of Physics, College of Science, Sultan Qaboos University, P.O. Box 36, Al-Khodh 123, Muscat, Sultanate of Oman
}

Received: 25 July 2018 / Accepted: 11 September 2018 / Published online: 25 September 2018

(C) The Author(s) 2018

\begin{abstract}
We study cosmological solutions in nonlocal teleparallel gravity or $f(T)$ theory, where $T$ is the torsion scalar in teleparallel gravity. This is a natural extension of the usual teleparallel gravity with nonlocal terms. In this work the phase space portrait proposed to describe the dynamics of an arbitrary flat, homogeneous cosmological background with a number of matter contents, both in early- and late-time epochs. The aim was to convert the system of the equations of the motion to a first order autonomous dynamical system and to find fixed points and attractors using numerical codes. For this purpose, firstly we derive effective forms of cosmological field equations describing the whole cosmic evolution history in a homogeneous and isotropic cosmological background and construct the autonomous system of the first order dynamical equations. In addition, we investigate the local stability in the dynamical systems called "the stable/unstable manifold" by introducing a specific form of the interaction between matter, dark energy, radiation and a scalar field. Furthermore, we explore the exact solutions of the cosmological equations in the case of a de Sitter spacetime. In particular, we examine the role of an auxiliary function called "gauge" $\eta$ in the formation of such cosmological solutions and show whether the de Sitter solutions can exist or not. Moreover, we study the stability issue of the de Sitter solutions both in vacuum and non-vacuum spacetimes. It is demonstrated that, for nonlocal $f(T)$ gravity, the stable de Sitter solutions can be produced even in vacuum spacetime.
\end{abstract}

\section{Introduction}

There has been found evidence for that in addition to the inflationary stage [1-5] in the early Universe, currently

\footnotetext{
a e-mail: bamba@sss.fukushima-u.ac.jp

be-mail: davood@squ.edu.om

ce-mail: mudhahir@squ.edu.om
}

the expansion of the Universe is also accelerating by various cosmological observations including Type Ia Supernovae $[6,7]$, cosmic microwave background (CMB) radiation [8-14], large scale structure [15,16], baryon acoustic oscillations (BAO) [17] as well as weak lensing [18]. We have two representative explanations for such a latetime cosmic acceleration. One approach is to introduce "dark energy" (DE) in the context of general relativity. The other approach is to consider the modification of gravity on the large scale (for reviews on not only DE problem but also modified gravity theories, see, for example, [1922]).

There is a possible candidate for a theory of gravitation alternative to general relativity, namely, teleparallel gravity, which is described by using the Weitzenböck connection [23-26]. In teleparallel gravity, there exists torsion. This is opposite to the case of general relativity, in which the LeviCivita connection is used. The torsion scalar $T$ represents the Lagrangian density of teleparallel gravity. It can be extended to a function of $T$, that is, $f(T)$ gravity (for a recent review, see, for instance, [27]). This idea is similar to that of $f(R)$ gravity [28-31], where $R$ is the scalar curvature. Inflation in the early Universe [32-34] and the late-time cosmic acceleration [35-38] can be realized in $f(T)$ gravity. Various cosmological and astrophysical considerations in $f(T)$ gravity have widely been performed [39-56]. It is well known that, in $f(T)$ gravity, the local Lorentz invariance is broken [57,58], and the relevant points in order have been discussed [5966].

On the other hand, in Ref. [67], there has been considered a way of modifying gravitation, the so-called nonlocal gravity, which comes from quantum effects. Furthermore, in order to unify inflation in the early Universe and the late-time accelerated expansion of the Universe, nonlocal gravity has been modified by adding an $f(R)$ term in Ref. [68]. In addition, a possible solution for the cosmological constant problem through the nonlocal property of gravitation [69] has been proposed. Moreover, a physical mech- 
anism by which a cosmological constant is screened in the framework of nonlocal gravity has been investigated [7072]. It has also been indicated that, in nonlocal gravity, there is the issue of ghosts [70]. Various aspects of nonlocal gravity have widely been explored [73-85] (for a recent review of nonlocal gravity, see, e.g., [102]). It is worth noting that the nonlocal term $\square T$ was first used in the framework of modified teleparallel gravity in Ref. [103] Furthermore, the nonlocal deformations of teleparallel gravity have been analyzed in Refs. $[104,105]$. This theory is called nonlocal $f(T)$ gravity, which can be considered as an extension of nonlocal general relativity to the Weitzenböck spacetime. It has been discussed that there is a possibility to distinguish teleparallel gravity from general relativity by future experiments detecting nonlocal effects. In this paper, we investigate exact cosmological solutions in nonlocal $f(T)$ gravity. We analyze the autonomous system of the first order dynamical equations by deriving effective forms of cosmological field equations in a homogeneous and isotropic cosmological background, describing the whole evolution history of the Universe. Moreover, we propose a specific form of the interaction between matter, dark energy, radiation and a scalar field and examine the local stability in the dynamical systems, which is called "the stable/unstable manifold". As a result, it is demonstrated that the system has a stable attractor. Furthermore, we study exact solutions of the cosmological equations in the case of de Sitter spacetime. Particularly, we explore the role of an auxiliary function, called "gauge", $\eta$, in the formation of such cosmological solutions and show whether the de Sitter solutions can exist or not in this scenario. In addition, we consider the stability problem of the de Sitter solutions both in vacuum and non-vacuum spacetimes and find that even in vacuum spacetime, the stable de Sitter solutions can be produced in the framework of nonlocal $f(T)$ gravity.

The organization of the paper is the following. In Sect. 2, we explain the framework of nonlocal $f(T)$ gravity. In Sect. 3, we explore the cosmological background and effective field equations in nonlocal $f(T)$ gravity. In Sect. 4, the interaction term and phase portrait are analyzed. In Sect. 5, the de Sitter solution is derived and its stability is examined in Sect. 6. Finally, conclusions are provided in Sect. 7.

\section{Formal framework of nonlocal $f(T)$ gravity}

Let us develop the formalism of nonlocal modified gravity with torsion $T$ in a same manner as the nonlocal $f(R)$ gravity is developed [68]. We suppose that the possible action for gravity with matter contents is given in terms of classical gauge invariant action as follows:

$$
S=\frac{1}{2 \kappa} \int \mathrm{d}^{4} x e T\left(f\left(\square^{-1} T\right)-1\right)+\int \mathrm{d}^{4} x e \mathcal{L}_{m},
$$

where $\kappa=8 \pi G, G$ is Newtonian gravitational constant, $\mathcal{L}_{m}$ is matter Lagrangian. To describe the geometry of spacetime in teleparallel gravity, the tetrad formalism is commonly used where the metric can be written in an orthogonal frame $e_{a}^{\mu}$, in a such manner that $g^{\mu \nu}=e_{a}^{\mu} e_{b}^{v} \eta^{a b}$, where Greek characters run from $\mu, v=0, \ldots, 3$, and the flat Minkowski metric is denoted by $\eta^{a b}$. Note that $e_{a}^{\mu} e_{v}^{a}=\delta_{v}^{\mu}$ and $\square^{-1}$ is considered as an integral over the entirely spacetime manifold. The local operator $\square=\nabla^{\mu} \nabla_{\mu}$ is called d'Alembert operator defined as $\square=e^{-1} \partial_{\alpha}\left(e \partial^{\alpha}\right)$, where $e=\operatorname{det}\left(e_{a}^{\mu}\right)=\sqrt{-\operatorname{det}\left(g_{\alpha \beta}\right)}$ and $T$ is the torsion scalar; it is defined in the same form as in $f(T)$ gravity.

It is always possible to reduce nonlocal theories to scalartensor equivalent theories and it is easy to do that for our model given in (1) using two auxiliary (nonghost) fields, $\phi=$ $\frac{1}{\square} T$ and $\xi=-\frac{1}{\square}\left(f^{\prime}(\phi) T\right)$. The reason that those fields are considered to be nonghost is that the norm of them, defined as $\|\phi\|=\int_{\Sigma}|\phi|^{2} e d^{4} x$, is always positive definite and never becomes complex as long as the metric and its torsion $T$ remain real numbers. As long as we work in Riemanninan manifolds, this condition will hold and we can safely use them as an appropriate set of auxiliary fields.

The new form for the reduced action is written as follows:

$$
\begin{aligned}
S= & \frac{1}{2 \kappa} \int \mathrm{d}^{4} x e\left[T(f(\phi)-1)-\partial_{\mu} \xi \partial^{\mu} \phi-\xi T\right] \\
& +\int \mathrm{d}^{4} x e \mathcal{L}_{m} .
\end{aligned}
$$

Note that in (3) the action function $f(\phi)$ is supposed to have any desired form. Formally if we take the case $\xi=1$ and $f(\phi)=2$ in the action of the theory given by Eq. (2), then the action is reduced to the one which is an equivalent of teleparallel gravity for $T \neq 0$. Classical tests for GR prove to be in very good agreement with observations. As a result it is very important to know whether this nonlocal teleparallel gravity has the GR limit or not. At the level of the action we already demonstrate it. By sufficiently well chosen function $f(\phi)$ we can recover GR as a limiting case.

The form of the equations of motion is presented in [104]:

$$
\begin{aligned}
& 2(1-f(\phi)+\xi)\left[e^{-1} \partial_{\mu}\left(e S_{a}{ }^{\mu \beta}\right)-E_{a}^{\lambda} T^{\rho}{ }_{\mu \lambda} S_{\rho}{ }^{\beta \mu}-\frac{1}{4} E_{a}^{\beta} T\right] \\
& \quad-\frac{1}{2}\left[\left(\partial^{\lambda} \xi\right)\left(\partial_{\lambda} \phi\right) E_{a}^{\beta}-\left(\partial^{\beta} \xi\right)\left(\partial_{a} \phi\right)-\left(\partial_{a} \xi\right)\left(\partial^{\beta} \phi\right)\right] \\
& \quad-2 \partial_{\mu}(\xi-f(\phi)) E_{a}^{\rho} S_{\rho}{ }^{\mu \nu}=\kappa \Theta_{a}^{\beta}, \\
& \square \xi+T f^{\prime}(\phi)=0, \\
& \square \phi-T=0 .
\end{aligned}
$$

Here the tensor $E_{\alpha}^{\beta}$ is defined through the variation of $e$ as follows: $\delta e=e E_{\alpha}^{\beta} e_{\beta}^{a}, \Theta_{a}^{\beta}$ is the energy-momentum 
tensor of matter contents defined by $\Theta_{a}^{\beta}=e^{-1} \frac{\delta\left(e \mathcal{L}_{m}\right)}{\delta e_{\beta}^{a}}$ and $\square \equiv e^{-1} \partial_{\mu}\left(e \partial^{\mu}\right)$.

In Ref. [104], the authors investigated cosmological data analysis by a suitably chosen function $f(\phi)=A \exp (n \phi)$ and later, in Ref. [105], using a Noether symmetry approach. In our paper we will fix $f(\phi)$ in another simple/adequate form in the next section.

\section{Cosmological background and effective field equations}

The aim of this section is to write the equations of motion for a cosmological background in the presence of matter fields in an effective form. Let us suppose that the nonsingular, physical metric of spacetime is given in the form of the Friedman-Lemaitre-Robertson-Walker (FLRW) metric, given by $\mathrm{d} s^{2}=\mathrm{d} t^{2}-a(t)^{2}\left(\mathrm{~d} x^{b} \mathrm{~d} x_{b}\right)$, where $b=$ $1,2,3$ is spatial coordinate and $a(t)$ is scale factor, measuring the expansion of the whole cosmological Universe as well as its acceleration/deceleration phase. The corresponding suitable, diagonal tetrad basis is given by $e_{\mu}^{a}=$ $\operatorname{diag}(1, a(t), a(t), a(t))$. The set of FLRW equations and the equations for the scalar fields are written as follows:

$$
\begin{aligned}
& 3 H^{2}(1+\xi-f(\phi))=\frac{1}{2} \dot{\phi} \dot{\xi} \\
& \quad+\kappa\left(\rho_{m}+\rho_{\Lambda}+\rho_{r}\right), \\
& \left(2 \dot{H}+3 H^{2}\right)(1+\xi-f(\phi))=-\frac{1}{2} \dot{\phi} \dot{\xi} \\
& \quad+2 H(\dot{\xi}-\dot{f}(\phi))-\kappa\left(p_{\Lambda}+p_{r}\right), \\
& \ddot{\xi}+3 H \dot{\xi}-6 H^{2} f^{\prime}(\phi)=0, \\
& \ddot{\phi}+3 H \dot{\phi}+6 H^{2}=0 .
\end{aligned}
$$

The matter energy-momentum tensor is given in terms of a diagonal tensors for matter, dark energy, and radiation as follows:

$$
\begin{aligned}
& \tau_{\mu}^{\nu}=e_{\mu}^{a} e_{b}^{\nu} \tau_{a}^{b}=\operatorname{diag}\left(\rho_{m}+\rho_{\Lambda}+\rho_{r},-p_{\Lambda}\right. \\
& \left.\quad-p_{r},-p_{\Lambda}-p_{r},-p_{\Lambda}-p_{r}\right)
\end{aligned}
$$

where $e_{\mu}^{a} e_{a}^{\mu}=\delta_{b}^{a}$ is the unit matrix. The matter budget of our model is the dark matter density $\rho_{m}$, the radiation field density $\rho_{r}$ and the scalar field density $\rho_{\phi}$. In order to preserve the acceleration expansion and the existence of late-time de Sitter cosmology we inserted a nonzero cosmological constant $\Lambda$ with energy density $\rho_{\Lambda}$. In an attempt to keep simplicity we assume that all matter contents are given in barotropic forms, where we define the equation of state $(\mathrm{EoS})$ parameter $w_{a}$ for each fluid component, namely matter, radiation and cosmological constant, and as a result for any component of matter field we have a linear EoS, i.e., $p_{a}=w_{a} \rho_{a}$. Here the Roman index $a$ refers to different matter contents.
Namely we denote it by $a=\{m, \Lambda, r\}$ where $m$ is for matter, $\Lambda$ is for DE and $r$ is for the radiation field. Note that neither $\phi$ nor $\xi$ are considered as the DE. The reason is that both fields play the role of auxiliary fields. We cannot guarantee that the fields $\phi, \xi$ will be ghost or not. Actually the appearance of ghost scalar fields in the nonlocal theories for gravity is an important issue and should be addressed adequately. For example in the nonlocal extensions of the GR, when the action is corrected by nonlocal terms $\frac{1}{\square} R$ or higher order terms, one must count the number of degrees of freedom of the localized form of the Lagrangian. Additionally, one needs to check the equivalence between local and nonlocal representations of the theory both at the action level and equation of motion levels. It is possible to make a categorization based on the first form of the auxiliary fields. Based on this classification we can find the number of algebraic constraints, which will limit our ability to write the local or nonlocal representations of theory. However, in nonlocal extensions of GR, as long as we have a linear term, we can ascertain the equivalence between frames. However, with higher order terms this equivalence is broken. That means in a general nonlocal GR when we have only curvature terms, our theory may suffer from ghosts. In nonlocal extensions of teleparallel gravity (TEGR), we can deduce the same as long as we develop the theory using a nonlocal action made by linear scalar torsion $T$, and, hence, no ghost will appear. That is because it was proved that the Einstein-Hilbert action of GR is dynamically equivalent to the TEGR at the level of the action as well as the equations of motion [106]. In our study with the nonlocal term which we will opt for in next paragraph the model will be ghost free. Although probably the scalar field $\phi$ will not be a ghost, still we do not have any strong reason to keep it as the only sector for the acceleration expansion in our model. For this reason we also keep the cosmological constant $\Lambda$ and its energy density $\rho_{\Lambda}$.

The first challenge is to choose one suitable form for $f(\phi)$. Note that $\square \xi=-f^{\prime}(\phi) \square \phi$. It is illustrative to expand this equation and write it in the following equivalent form:

$\square(\xi+f(\phi))=f^{\prime \prime}(\phi) \square \phi$.

Here, we suppose that $f^{\prime \prime}(\phi)=0$. Thus one suitable class of models is

$$
f(\phi)=A \phi+B .
$$

Note that for $A=0, B=2$ the results reduce to GR as a limiting case. Note that now $\square(\xi+f(\phi))=0$, and we have the freedom to take $\xi+f(\phi)=\Psi$, where $\Psi$ is a harmonic function over $\mathcal{R}^{4}$. A possible option is to consider $\Psi=2 A \phi$; consequently the set of Eqs. (6) and (7) are simply written in the following forms:

$$
3 H^{2}=\frac{\frac{1}{2} A \dot{\phi}^{2}+\kappa\left(\rho_{m}+\rho_{\Lambda}+\rho_{r}\right)}{1-2 B},
$$




$$
\begin{aligned}
& 2 \dot{H}+3 H^{2}=-\frac{\frac{1}{2} A \dot{\phi}^{2}+\kappa\left(p_{\Lambda}+p_{r}\right)}{1-2 B}, \\
& \ddot{\xi}+3 H \dot{\xi}-6 A H^{2}=0, \\
& \ddot{\phi}+3 H \dot{\phi}+6 H^{2}=0 .
\end{aligned}
$$

Here the parameter $B$ measures the difference between TEGR and nonlocal theory, respectively. Because we will study time evolution of the energy densities, it is adequate to rewrite the cosmological equation, presented in Eq. (12), in the following forms:

$$
\begin{aligned}
3 H^{2}= & \frac{\rho_{\phi}}{1-2 B}+\frac{\rho_{m}}{1-2 B} \\
& +\frac{\rho_{\Lambda}}{1-2 B}+\frac{\rho_{r}}{1-2 B} ;
\end{aligned}
$$

in the above equation, we have defined

$$
\rho_{\phi} \equiv A \dot{\phi}^{2} \text {. }
$$

Note that the other density functions cannot be written explicitly in terms of the scale factor $a$ or scalar fields, until we present the continuity equations for all the matter components. In our scenario we assumed that different matter contents interact with each other through some interaction forms which will be presented in next section.

\subsection{Hartman-Grobman linearizion theorem}

To investigate the phase space analysis one needs to reduce the system of equations to an autonomous system of first order differential equations in the form $\frac{\mathrm{d} \vec{X}}{\mathrm{~d} N}=f(\vec{X})$, where $N$ plays the role of time and $\vec{X}$ is a vector field with density functions as components. In much work, various aspects of dynamical systems in the cosmology of modified gravity are discussed [89].

The Hartman-Grobman linearizion theorem provides a powerful technique to study the local stability and the portrait of the phase space, when we have a set of hyperbolic fixed points. Let $\vec{X}(t) \in \mathcal{R}^{n}$ be a nontrivial solution to the following system of first order differential equations, called the flow:

$$
\frac{\mathrm{d} \vec{X}}{\mathrm{~d} t}=g(\vec{X}) .
$$

Here $g(\vec{X})$ is a locally Lipschitz, one-to one continuous map $g: \mathcal{R}^{n} \rightarrow \mathcal{R}^{n}$. Let $\vec{X}_{*}$ denote the location of the fixed points of the dynamical system (18), and the corresponding Jacobian matrix, which we denote $\mathcal{J}(g)$, be equal to

$$
(\mathcal{J})_{i j}=\left[\frac{\partial \mathrm{g}_{i}}{\partial X_{j}}\right] .
$$

In order to have stable fixed points for system (18) it is enough to set all eigenvalues of the Jacobian matrix so that $\lambda_{i}$ sat- isfies $\operatorname{Re}\left(\lambda_{i}\right) \neq 0$. The Hartman theorem predicts the existence of a homeomorphism $\mathcal{F}: U \rightarrow \mathcal{R}^{n}$, where $U$ is an open neighborhood of $\vec{X}_{*}$, such that $\mathcal{F}\left(\vec{X}_{*}\right)$ is defined. The homeomorphism generates a flow $\frac{\mathrm{d} h(u)}{\mathrm{d} t}$, which is

$\frac{\mathrm{d} h(u)}{\mathrm{d} t}=\mathcal{J} h(u)$.

It is proved that (20) is a topologically conjugate flow to the one system given in Eq. (18).

\subsection{Building the cosmological autonomous system of equations}

Now we study a model of interacting matter contents, where the continuity equation for each energy density $\rho_{a}$ is given by the following form:

$$
\dot{\rho}_{a}+3 H\left(1+w_{a}\right) \rho_{a}=\Gamma_{a},
$$

where we use $a=\{m, \Lambda, r, \phi\}$ and $\Gamma_{a}$ is the interaction function given by the general form $\Gamma_{a}=\Gamma_{a}\left(\Omega_{m}, \Omega_{\Lambda}, \Omega_{r}, \Omega_{\phi}\right)$ satisfying $\Sigma_{a=1}^{4} \Gamma_{a}=0$. In $f(T)$ gravity, such interacting models are widely studied in the literature, namely [50-109]. In Ref. [110] the authors showed that the total gravitational energy is transferred from dark matter $\rho_{m}$ to dark energy $\rho_{\Lambda}$, and the cosmological coincidence problem in the LambdaCold Dark Matter $(\Lambda \mathrm{CDM})$ model is slightly assuaged.

In comparison to the matter, DE and radiation energy densities, let us define an auxiliary scalar energy density as

$\Omega_{\phi}=\frac{A \dot{\phi}^{2}}{3 H^{2}}$.

It is important to mention here that the auxiliary field $\phi$ is not a physical field. Consequently the kinetic term could be treated as a tachyonic field as well as pressureless dust matter. In this paper we consider $w_{\phi}$ as a free parameter to be adjusted using observational data.

In this case we can write the following equation for the ratio between pressure and density, called the effective EoS equation:

$$
\frac{2 \dot{H}}{3 H^{2}}=\frac{1}{2 B-1}\left(\Omega_{\phi}+\Omega_{m}+\left(1+w_{\Lambda}\right) \Omega_{\Lambda}+\left(1+w_{r}\right) \Omega_{r}\right),
$$

where we prescribed the form of $f(\phi)$ as given in Eq. (11) and we supposed that $w_{m}=w_{\phi}=0, w_{r}=\frac{1}{3}, w_{\Lambda} \in\left(-1,-\frac{1}{3}\right)$.

It is easy to rewrite (21) using the definition of

$\Omega_{a}=\frac{\kappa \rho_{a}}{3 H^{2}}$

in the following set of first order differential equations where we used (22): 


$$
\begin{aligned}
\frac{\mathrm{d} \Omega_{a}}{\mathrm{~d} N}= & \frac{\kappa \Gamma_{a}}{3 H^{3}}-\Omega_{a}\left(\left(1+w_{a}\right)+\frac{3}{2 B-1}\left(\Omega_{\phi}\right.\right. \\
& \left.\left.+\Omega_{m}+\left(1+w_{\Lambda}\right) \Omega_{\Lambda}+\left(1+w_{r}\right) \Omega_{r}\right)\right) .
\end{aligned}
$$

Recall $a=\{m, \Lambda, r, \phi\}$ and we use the slow-roll variable $N=\log \left(\frac{a}{a_{0}}\right)=-\ln (1+z)$ (the derivatives will be taken with respect to $N$ ) and $z$ is the redshift. This is an autonomous system and should be analyzed in the vicinity of critical points where $\left.\frac{d \Omega_{a}}{d N}\right|_{c}=0$ using the techniques developed in Sect. 3.1.

In terms of the variables (23) the Friedmann equation (12) becomes the restriction

$$
\Omega_{m}+\Omega_{\Lambda}+\Omega_{r}+\Omega_{\phi}=1-2 B .
$$

Note that due to the interaction term in the model, the density parameters $\Omega_{m}, \Omega_{\Lambda}, \Omega_{r}, \Omega_{\phi}$ should be interpreted very strictly as effective density parameters. We mention here that the above constraint guaranteed the existence of possible cosmological attractors, because actually the shape of the density functions remains typically the same and the full 4dimensional configuration space constructed using density functions defines a shape invariant manifold and it defines the attractor solution in the dynamical system.

The effective EoS for system is defined by

$$
\begin{aligned}
w_{\mathrm{eff}}= & \frac{p_{\text {tot }}}{\rho_{\text {tot }}}=-1-\frac{1}{2 B-1}\left(\Omega_{\phi}\right. \\
& \left.+\Omega_{m}+\left(1+w_{\Lambda}\right) \Omega_{\Lambda}+\left(1+w_{r}\right) \Omega_{r}\right) .
\end{aligned}
$$

\section{Interaction term and phase portrait}

The general linear dependent model for the interaction could be in the following form:

$$
\Gamma_{a}=\frac{3 H^{3}}{\kappa} \Sigma_{b=1}^{4} \alpha_{a b} \Omega_{b} .
$$

Some criticism as regards interacting models of DE are in order; however, the thermal properties of this model in various theories of gravity have been discussed in the literature [111-115]. Furthermore, in Ref. [116], the authors proposed a systematic scheme to construct the interaction form $\Gamma_{a}$ in a self consistent manner both in the perturbed form and in the background. They proved that in the perturbation formalism, there are possible ways to break the degeneracy between the interaction, DE EoS and DM abundance.

With this choice, the system of equation (24) is written in the following form:

$$
\begin{aligned}
\frac{\mathrm{d} \Omega_{a}}{\mathrm{~d} N}= & \Sigma_{b=1}^{4} \alpha_{a b} \Omega_{b}-\Omega_{a}\left(\left(1+w_{a}\right)+\frac{3}{2 B-1}\left(\Omega_{\phi}\right.\right. \\
& \left.\left.+\Omega_{m}+\left(1+w_{\Lambda}\right) \Omega_{\Lambda}+\left(1+w_{r}\right) \Omega_{r}\right)\right) .
\end{aligned}
$$

Let us study a class of these models where the DE interacts with both matter $\Omega_{m}$ and scalar field components.
Based on our former notation given in (27), our interaction model is parametrized as follows:

$$
\alpha_{m \Lambda}=-6 b, \alpha_{\Lambda \Lambda}=\alpha_{r \Lambda}=\alpha_{\phi \Lambda}=2 b .
$$

The autonomous system of first order differential equations for density functions is written in the following forms:

$$
\begin{aligned}
\frac{\mathrm{d} \Omega_{m}}{\mathrm{~d} N}= & -6 b \Omega_{\Lambda}-\Omega_{m}\left(1+\frac{3}{2 B-1}\left(\Omega_{\phi}\right.\right. \\
& \left.\left.+\Omega_{m}+\left(1+w_{\Lambda}\right) \Omega_{\Lambda}+\left(1+w_{r}\right) \Omega_{r}\right)\right) \equiv f_{1}, \\
\frac{\mathrm{d} \Omega_{\Lambda}}{\mathrm{d} N}= & 2 b \Omega_{\Lambda}-\Omega_{\Lambda}\left(\left(1+w_{\Lambda}\right)+\frac{3}{2 B-1}\left(\Omega_{\phi}\right.\right. \\
& \left.\left.+\Omega_{m}+\left(1+w_{\Lambda}\right) \Omega_{\Lambda}+\left(1+w_{r}\right) \Omega_{r}\right)\right) \equiv f_{2}, \\
\frac{\mathrm{d} \Omega_{r}}{\mathrm{~d} N}= & 2 b \Omega_{\Lambda}-\Omega_{r}\left(\frac{4}{3}+\frac{3}{2 B-1}\left(\Omega_{\phi}\right.\right. \\
& \left.\left.+\Omega_{m}+\left(1+w_{\Lambda}\right) \Omega_{\Lambda}+\left(1+w_{r}\right) \Omega_{r}\right)\right) \equiv f_{3}, \\
\frac{\mathrm{d} \Omega_{\phi}}{\mathrm{d} N}= & 2 b \Omega_{\Lambda}-\Omega_{\phi}\left(\left(1+w_{\phi}\right)+\frac{3}{2 B-1}\left(\Omega_{\phi}\right.\right. \\
& \left.\left.+\Omega_{m}+\left(1+w_{\Lambda}\right) \Omega_{\Lambda}+\left(1+w_{r}\right) \Omega_{r}\right)\right) \equiv f_{4} .
\end{aligned}
$$

These equations are related to the dynamics and the interaction form, characterizing the main properties of our model.

\subsection{The critical (fixed) points}

We stress here that the high dimensionality of the phase space, where the system is described using dynamical systems presented in the previous section, restricts us to having an effective graphical description of the phase space, and thus we will focus our investigations only on the analytical results.

To make the dynamical analysis we first need to find the critical(fixed) points of the system by setting the left hand side of Eqs. (30)-(33) to zero. Then we use the Hartman theorem to find the type and stability of each point [117-120].

The location of the fixed points $P=\left(\Omega_{m}, \Omega_{\Lambda}, \Omega_{r}, \Omega_{\phi}\right)$ and their corresponding eigenvalues of the dynamical system are in the following table, where the stability of the fixed points is determined by evaluating the eigenvalues of the Jacobian matrix associated with the system:

where

$$
\begin{aligned}
x_{2}= & \left(-9 w_{\Lambda}^{4}+\left(54 b+9 w_{\phi}-6\right) w_{\Lambda}^{3}\right. \\
& +\left(-108 b^{2}+\left(-36 w_{\phi}+30\right) b+6 w_{\phi}+3\right) w_{\Lambda}^{2} \\
& +\left(72 b^{3}+\left(36 w_{\phi}-48\right) b^{2}-3 w_{\phi}\right) w_{\Lambda} \\
& \left.+24 b^{3}+\left(-24 w_{\phi}-12\right) b^{2}-12 b w_{\phi}\right)
\end{aligned}
$$




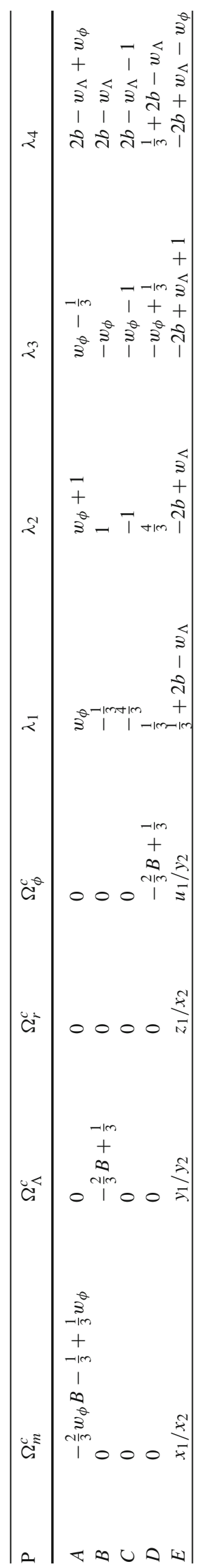

$$
\begin{aligned}
y_{2}= & \left(-3 w_{\Lambda}^{4}+\left(18 b+3 w_{\phi}-2\right) w_{\Lambda}^{3}\right. \\
& +\left(-36 b^{2}+\left(-12 w_{\phi}+10\right) b+2 w_{\phi}+1\right) w_{\Lambda}^{2} \\
& +\left(24 b^{3}+\left(12 w_{\phi}-16\right) b^{2}-w_{\phi}\right) w_{\Lambda} \\
& +8 b^{3}+\left(-8 w_{\phi}-4\right) b^{2} \\
& \left.-4 b w_{\phi}\right) x_{1}=x(2 b)\left(6 b-3 w_{\Lambda}+1\right)\left(2 b-w_{\Lambda}\right) \\
y_{1}= & x(-2 b)\left(6 b-3 w_{\Lambda}+1\right)\left(2 b-w_{\Lambda}+w_{\phi}\right) \\
z_{1}= & x\left(2 b-w_{\Lambda}\right)\left(2 b-w_{\Lambda}+w_{\phi}\right)\left(6 b-3 w_{\Lambda}+1\right) \\
u_{1}= & \left.x(2 b)\left(2 b-w_{\Lambda}\right)\right)\left(2 b-w_{\Lambda}+w_{\phi}\right) \\
& \text { and } \\
x= & \left(2 b-w_{\Lambda}-1\right)(2 B-1) .
\end{aligned}
$$

The corresponding Jacobian matrix, which we denote $\mathcal{J}(g)$, is equal to

$\left[\begin{array}{llll}\frac{\partial f_{1}}{\partial \Omega_{m}} & \frac{\partial f_{1}}{\partial \Omega_{\Lambda}} & \frac{\partial f_{1}}{\partial \Omega_{r}} & \frac{\partial f_{1}}{\partial \Omega_{\phi}} \\ \frac{\partial f_{2}}{\partial \Omega_{m}} & \frac{\partial f_{2}}{\partial \Omega_{\Lambda}} & \frac{\partial f_{2}}{\partial \Omega_{r}} & \frac{\partial f_{2}}{\partial \Omega_{\phi}} \\ \frac{\partial f_{3}}{\partial \Omega_{m}} & \frac{\partial f_{3}}{\partial \Omega_{\Lambda}} & \frac{\partial f_{3}}{\partial \Omega_{r}} & \frac{\partial f_{3}}{\partial \Omega_{\phi}} \\ \frac{\partial f_{4}}{\partial \Omega_{m}} & \frac{\partial f_{4}}{\partial \Omega_{\Lambda}} & \frac{\partial f_{4}}{\partial \Omega_{r}} & \frac{\partial f_{4}}{\partial \Omega_{\phi}}\end{array}\right]$.

The eigenvalues and their stability for each point are written as follows.

- Stability for point $A$ : The necessary and sufficient condition to have $A_{1}$ as a stable fixed point for system is that all eigenvalues of the Jacobian matrix $\lambda_{i}$ must satisfy $\operatorname{Re}\left(\lambda_{i}\right) \neq 0$, i.e.,

$$
\begin{aligned}
& w_{\Lambda} \leq 2 b-1, \quad b<\frac{1}{3} w_{\Lambda}-\frac{1}{3} w_{\phi}, \\
& w_{\phi}<-1, \quad 2 b+\frac{1}{3}<w_{\Lambda}, \\
& w_{\Lambda} \leq 2 b+\frac{1}{3}, \quad w_{\phi}<-1, \quad 2 b-1<w_{\Lambda} .
\end{aligned}
$$

From these it is found that the stability occurs at $2 b-1<$ $w_{\Lambda} \leq 2 b+\frac{1}{3}$.

$w_{\phi}<-1$. The corresponding effective EoS behaves like $w_{\text {eff }}=w_{\phi}$. Depending on the $w_{\phi}$, the EoS evolves from larger than -1 to less than -1 , that is, it crosses the phantom divide line of $w_{\text {eff }}=-1$.

- Stability for point $B$

This is an unstable critical point and the corresponding effective EoS, $w_{\text {eff }}=w_{\Lambda}$, is always larger than -1 and it crosses the phantom divide line when $w_{\Lambda}=-1$.

- Stability for point $C$ :

The stability condition is $-1<w_{\phi}, 2 b-1<w_{\phi}$. Consequently $C$ can be stable conditionally. The corresponding effective EoS is $w_{\text {eff }}=-1$; it is located at the crossings of the phantom divide line. 
- Stability for point $D$ :

We obviously conclude that it is unstable. The corresponding effective EoS is given by $w_{\text {eff }}=0$ and is always larger than -1 and it cannot cross the phantom divide line.

- Stability for point $E$ :

The point is stable conditionally only and only if $w_{\Lambda}<$ $2 b-1, \frac{1}{2} w_{\Lambda}-\frac{1}{2} w_{\phi}<b$.

\section{Cosmography}

The following types of observational data are commonly used to study cosmography.

SNe Ia: Type Ia supernovae ( $\mathrm{SNe}$ Ia) or the latest "joint light curves" (JLA) sample [121], comprised of 740 type Ia supernovae in the redshift range $0.01 \leq z \leq 1.30$.

BAO: The baryon acoustic oscillations (BAOs) [122-125] (see Table I of [126]).

$\mathbf{C C}+\mathrm{H}_{0}$ : The cosmic chronometer (CC) data set in the redshift range $0<z<2$ [127]. In $f(T)$ gravity cosmography is introduced and investigated in detail in Ref. [128].

In Fig. 1, we plot the time evolution of the density functions for $b=0.5,0.7,0.9, w_{\Lambda}=-1 / 3, w_{\phi}=0$, where the horizontal axis shows $\log (1+z)$ and the vertical axis shows the value of the density functions. We can observe that the density functions of matter, $\Omega_{m}$, and the cosmological constant $\Omega_{\Lambda}$ increase in time, whereas the density functions of radiation, $\Omega_{r}$ and $\Omega_{\phi}$, decrease in time. For low redshift values, $0<z<0.1$, the densities $\Omega_{\phi}, \Omega_{r}$ are monotonically increasing functions, but the matter and cosmological constant density functions decrease. At the present redshift $z \sim 0, \Omega_{\phi} \sim \Omega_{r}$ are negligible in comparison to the matter and cosmological constant densities. This confirms the remarkable observation that the scalar field $\phi$ cannot play the role of DE. So, the density of the scalar field is almost negligible at the present time. At distinct values of the redshift, shown as $z^{*}$, the densities of matter, radiation, and scalar field become equal, i.e. $\Omega_{m} \sim \Omega_{r} \sim \Omega_{\phi}$. This occurs at $z^{*} \approx 0.1$. Furthermore, there is an era when $z^{\dagger} \sim 0.4$, in which $\Omega_{m} \sim \Omega_{\lambda}$, showing another equilibrium among matter and cosmological constant. These behaviors of the density functions are compatible with the observations.

\subsection{Observation of a type of deceleration to acceleration} phase transition

The deceleration parameter $q$ is defined as

$q=-1-\frac{\dot{H}}{H^{2}}$.

If the expansion of the Universe is decelerating, then $q>-1$, while if the cosmic expansion is accelerating, then $q<-1$. In our model, using (22), we obtain

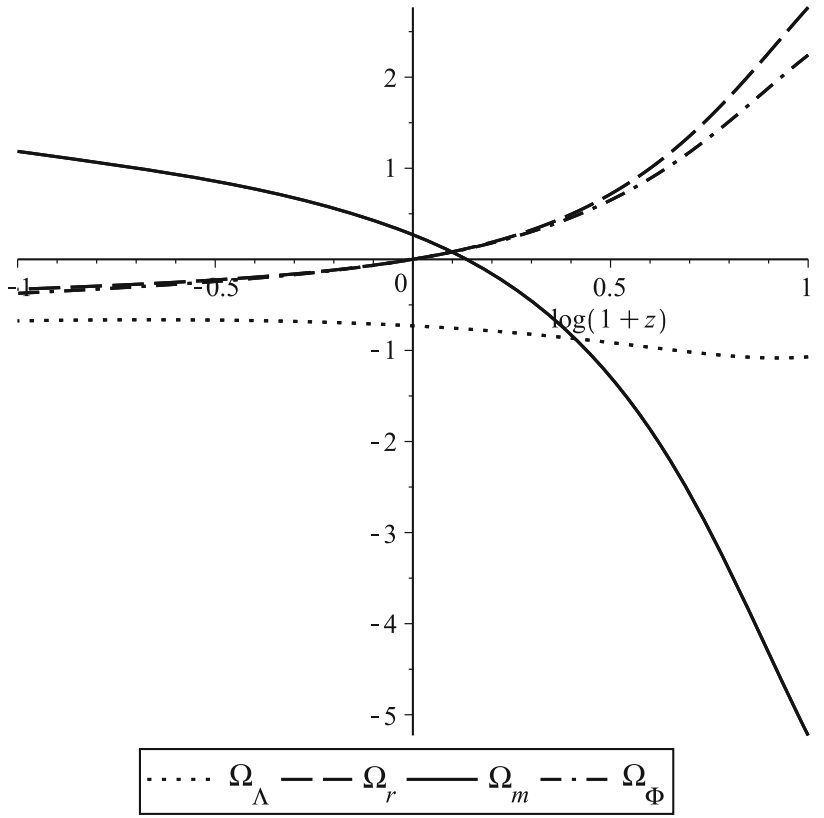

Fig. 1 Numerical solutions for density functions for the set of parameters $b=0.5, w_{\Lambda}=-1 / 3 ; w_{\phi}=0$. Here, the horizontal axis shows $\log (1+z)$ and the vertical axis shows the value of the density functions

$$
\begin{aligned}
q= & -1-\frac{3}{2(2 B-1)}\left(\Omega_{\phi}+\Omega_{m}\right. \\
& \left.+\left(1+w_{\Lambda}\right) \Omega_{\Lambda}+\left(1+w_{r}\right) \Omega_{r}\right) .
\end{aligned}
$$

We find numerical solutions by using $h=0.7127_{-0.015}^{+0.013}$ $\mathrm{km} / \mathrm{s} / \mathrm{Mpc}, \Omega_{\Lambda}=0.7018_{-0.02}^{+0.018}$, and $\Omega_{m 0}=0.2981_{-0.018}^{+0.02}$, with $\chi_{\min }^{2}=707.4, H_{0}=73.24 \pm 1.74 \mathrm{~km} / \mathrm{s} / \mathrm{Mpc}$. In Fig. 2, we depict the time evolution of the deceleration parameter $q$ for $b=0.5,0.7,0.9$. Here, the horizontal axis shows $\log (1+$ $z$ ) and the vertical axis shows the value of $q$. All of the curves meet. From Fig. 2, it is found that in the past for lower values of redshift, the value of $q$ evolved from larger than -1 to less than -1 , namely, the expansion phase of the Universe changed from deceleration to acceleration. This is consistent with the observations.

\subsection{Effective equation of state (EoS) of the Universe}

Effective EoS of the Universe was defined in Eq. (26). We note that in the DE dominated stage, the value of the EoS of $\mathrm{DE}$ can be regarded as the effective EoS of the Universe $w_{\text {eff }}$.

In Fig. 3, we show the time evolution of the effective EoS $w_{\text {eff }}$ for $b=0.5,0.7,0.9$, where the horizontal axis shows $\log (1+z)$ and the vertical axis shows the value of $w_{\text {eff. }}$. From Fig. 3 , it is found that for low redshift values, the value of $w_{\text {eff }}$ became less than $-\frac{1}{3}$ and therefore the cosmic expansion phase of the Universe changed from deceleration to acceleration. It is also seen that in our model, the 


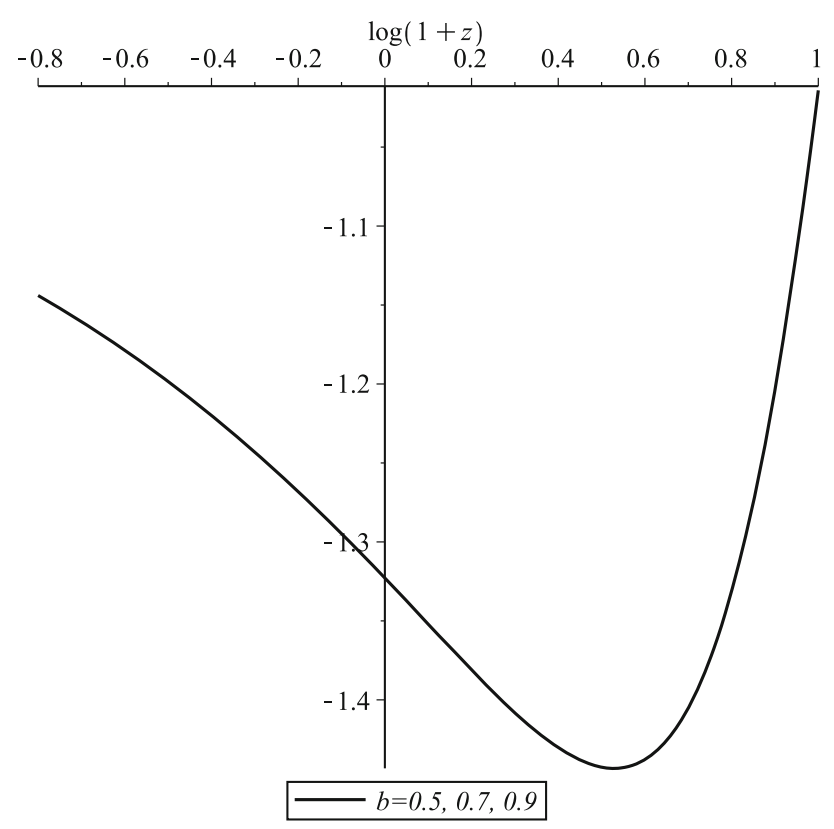

Fig. 2 Deceleration parameter $q$ for $b=0.5,0.7,0.9$. All of the curves meet. Here, the horizontal axis shows $\log (1+z)$ and the vertical axis shows the value of $q$

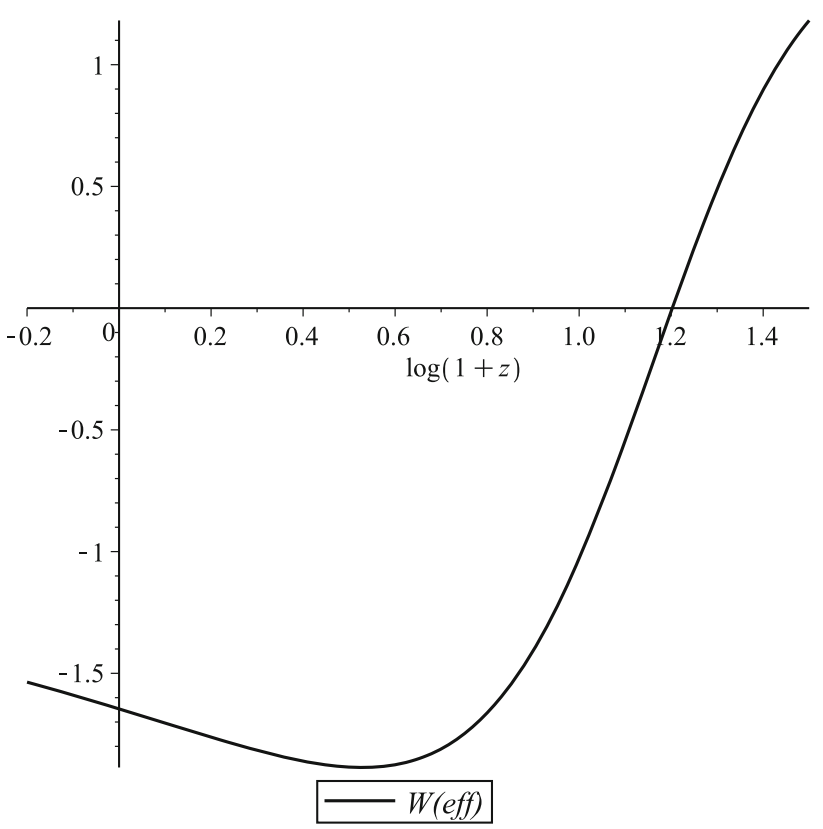

Fig. 3 Effective EoS $w_{\text {eff }}$ for $b=0.5, b=0.7, b=0.9$. The three curves coincides. Here, the horizontal axis shows $\log (1+z)$ and the vertical axis shows the value of $w_{\text {eff }}$

value of $w_{\text {eff }}$ evolves from larger than -1 to less than -1 ; that is, it crosses the phantom divide line of $w_{\text {eff }}=-1$. The value of the EoS parameter at the present redshift is around $w_{\text {eff }} \sim-1.6<-1$, showing an acceleration expansion beyond the phantom line.

\section{6 de Sitter solution}

Cosmological models usually have a de Sitter (dS) solution where the Hubble parameter is constant (or almost constant in inflationary scenarios) with $H=H_{0}$ as trivial solution. In GR such a solution (used in an inflationary mechanism as well as in late-time cosmology) becomes accessible when the dominant energy density $\rho \approx \rho_{0}$, which means that to have the dS case we need matter fields with very slowly varying energy density. It is not possible to find a dS solution as an empty space solution in GR. But in modified gravity because of the geometrical terms (curvature $\mathcal{R}$ or torsion $T$ ) it will be possible to find $\mathrm{dS}$ as an (almost) exact solution for the field equations. In forthcoming sections, we look for a dS solution both in empty and matter contents cases in model defined by Eqs. (6) and (7).

Let us firstly perform a little investigation on the equations of motion. As a result of the continuity equation, we have two additional Klein-Gordon like dissipative equations (21) in the noninteracting case, and we can find equations of motion for $\phi, \xi$. If we suppose that in (11), $B=0, A=-1$, they are written as follows:

$$
\begin{aligned}
& \ddot{\phi}+3 H \dot{\phi}+6 H^{2}=0, \\
& \ddot{\xi}+3 H \dot{\xi}+6 H^{2}=0 .
\end{aligned}
$$

Note that always with $f(\phi)=-\phi$, we have $\nabla^{\mu} \nabla_{\mu}(\phi-\xi)=$ 0 , and we have "gauge freedom" to write the fields $\phi, \xi$ as follows:

$$
\phi-\xi=\eta(t) .
$$

An exact solution for $\eta$, in FLRW background, is given by

$$
\eta(t)=\int \frac{\eta_{0}}{a(t)^{3}} \mathrm{~d} t .
$$

Note that $\dot{\eta}(t) \sim \rho_{m}(t)$. In this case we can interpret $\dot{\eta}(t)$ as a cold dark matter density. It is possible to take $\eta_{0}=0$ or $\eta_{0} \neq 0$. We will study both cases in next subsections.

\subsection{Empty spacetime}

To find an exact (almost exact) dS solution for a system given by (6), (7), (38) and (39) in vacuum, let us relax all matter contents, to make spacetime empty (we will never consider quantum fluctuations in this approach). Furthermore, we set $H=H_{0}$ for the dS case.

\subsubsection{Case $\eta(t)=0$ :}

When $\eta=0, \phi=\xi$. Thus the system (38), (39), and (22) is reduced to the following system:

$$
\frac{H_{0}}{\dot{\phi}}=\frac{1}{4},
$$




$$
\ddot{\phi}+3 H_{0} \dot{\phi}+6 H_{0}^{2}=0 \text {. }
$$

From the first equation we find $\phi(t)=4 H_{0} t+\phi_{0}$. If we substitute it in the second equation we obtain $H_{0}=0$. In $f(T)$ gravity, stability for the Einstein Universe is well studied in [129].

This is just an Einstein static Universe and it proves that no dS solution may exist.

Let us check whether this solution is stable or not. We make a perturbation around the solution given by $(H, \phi)=$ $\left(0, \phi_{0}\right)$. The equation is given by the following:

$$
\delta \ddot{\phi}+3 \dot{\phi} \delta H+3 H \delta \dot{\phi}+12 H \delta H=0,
$$

and substituting the zeroth order solution we find

$$
\delta \ddot{\phi}=0 \text {. }
$$

The exact solution for the perturbation function is

$$
\delta \phi=a t+b .
$$

When $t \rightarrow \infty$, we clearly observe that the perturbation is growing linearly and consequently the system becomes unstable under infinitesimal field and background perturbations.

\subsubsection{Case $\eta(t) \neq 0$}

When $\eta=\int \frac{\eta_{0}}{a(t)^{3}} \mathrm{~d} t$, we have $\dot{\phi}-\dot{\xi}=\frac{\eta_{0}}{a(t)^{3}}$. Thus the system (38), (39), and (22) is reduced to the following system:

$$
\begin{aligned}
& \frac{1}{2 H_{0}}=\frac{1}{\dot{\phi}}+\frac{1}{\dot{\xi}}, \\
& \dot{\phi}-\dot{\xi}=\frac{\eta_{0}}{a(t)^{3}} .
\end{aligned}
$$

Note that in the dS phase, $a(t)=a_{0} e^{H_{0} t}$. An exact solution for the field pair $\phi, \xi$ is given as follows:

$$
\begin{aligned}
\phi_{0}(t)= & C_{2}+2 H_{0} t-\frac{\eta_{0}}{6 a_{0}^{3} H_{0}} e^{-3 H_{0} t} \\
& +\frac{1}{6 H_{0} a_{0}^{3}}\left(\Delta-4 a_{0}^{3} \sqrt{H_{0}} \tanh ^{-1}\left(\frac{\Delta}{4 a_{0}^{3} \sqrt{H_{0}}}\right)\right), \\
\xi_{0}(t)= & C_{2}+C_{1}+2 H_{0} t+\frac{\eta_{0}}{6 a_{0}^{3} H_{0}} e^{-3 H_{0} t} \\
& +\frac{1}{6 H_{0} a_{0}^{3}}\left(\Delta-4 a_{0}^{3} \sqrt{H_{0}} \tanh ^{-1}\left(\frac{\Delta}{4 a_{0}^{3} \sqrt{H_{0}}}\right)\right) .
\end{aligned}
$$

Here $C_{1}, C_{2}$ are arbitrary integration constants and $\Delta=$ $16 H_{0} a_{0}^{6}+a_{0}^{3} H_{0}^{2} e^{-6 H_{0} t}$. The solutions given in (50) and (51) are exact solutions for the $\mathrm{dS}$ phase of our model under study. Let us study its stability under perturbations in a time representation of fields and backgrounds. In Sect. 7.1 another, equivalent analysis using slow-roll coordinate $N$ will be introduced.

The perturbation of the field equations (38), (39) and Eq. (7) around the solution given by $(H, \phi, \xi)=\left(H_{0}, \phi_{0}, \xi_{0}\right)$ substituted in Eqs. (50) and (51) is given by the following system of equations:

$$
\begin{aligned}
& \delta \ddot{\phi}+3 \dot{\phi}_{0} \delta H+3 H_{0} \delta \dot{\phi}+12 H_{0} \delta H=0, \\
& \delta \ddot{\xi}+3 \dot{\xi}_{0} \delta H+3 H_{0} \delta \dot{\xi}+12 H_{0} \delta H=0, \\
& 2 \delta \dot{H}+4 \phi_{0} \delta \dot{H}+\left(\dot{\xi}_{0}-2 H_{0}\right) \delta \dot{\phi}+ \\
& \left(\dot{\phi}_{0}-2 H_{0}\right) \delta \dot{\xi}-2\left(\dot{\xi}_{0}+\dot{\phi}_{0}\right) \delta H=0 .
\end{aligned}
$$

It is hard to find exact solutions for the perturbation functions and we do not discuss all this. Note that, asymptotically, $\phi \sim \xi \approx 2 H_{0} t$; consequently we can find the following solutions, which are valid only when $t \rightarrow \infty$ :

$$
\delta \phi \sim \delta \xi \approx\left(H_{0} t\right)^{2}, \quad \delta H \approx H_{0} t
$$

We conclude that the $\mathrm{dS}$ case behaves as an unstable phase in our model.

\subsection{Case of matter contents}

Now we study exact solutions in the dS phase when $\rho_{a} \neq 0$. As a general case we consider the model given in (6) and (7) for the general densities $\rho_{m}=\rho_{m}^{0} a(t)^{-3}$.

\subsubsection{Case $\eta(t)=0$}

When $\eta=0, \phi=\xi$, the system (22), (38), and (39) is reduced to the following system:

$$
\begin{aligned}
& \dot{\phi}^{2}-4 H_{0} \dot{\phi}+\kappa \rho_{m}^{0} a(t)^{-3}=0, \\
& \ddot{\phi}+3 H_{0} \dot{\phi}+6 H_{0}^{2}=0 .
\end{aligned}
$$

The exact solutions show that $H_{0}=0$ is the only possible solution. Thus, similar to the empty case, still we just have an Einstein static Universe.

If we perturb the system around the solutions given above, we have the following system of equations:

$$
\begin{aligned}
& \delta \ddot{\phi}=0, \\
& 2\left(1+2 \phi_{0}\right) \delta \dot{H}-\delta \rho_{m}(t)=0,
\end{aligned}
$$

where $\phi_{0}=\mathrm{C}$ is a constant. The system is clearly asymptotically unstable; consequently, no stable static Einstein Universe exists.

\subsubsection{Case $\eta(t) \neq 0$}

When $\eta=\int \frac{\eta_{0}}{a(t)^{3}} \mathrm{~d} t$, the system (22), (38), and (39) is reduced to the following system:

$$
\begin{aligned}
& \dot{\phi} \dot{\xi}-2 H_{0}(\dot{\phi}+\dot{\xi})+\kappa \rho_{m}^{0} a(t)^{-3}=0, \\
& \dot{\phi}-\dot{\xi}=\frac{\eta_{0}}{a(t)^{3}} .
\end{aligned}
$$

The exact solutions are given in terms of first integrals:

$$
\dot{\phi}=2 H_{0}+\frac{\eta_{0}}{2 a(t)^{3}}
$$




$$
\begin{aligned}
& -\frac{1}{2} \sqrt{\eta_{0} a(t)^{-6}-4 \kappa \rho_{m}^{0} a(t)^{-3}+16 H_{0}^{2}}, \\
\dot{\xi}= & 2 H_{0}-\frac{\eta_{0}}{2 a(t)^{3}} \\
& -\frac{1}{2} \sqrt{\eta_{0} a(t)^{-6}-4 \kappa \rho_{m}^{0} a(t)^{-3}+16 H_{0}^{2}} .
\end{aligned}
$$

Therefore we have a $\mathrm{dS}$ solution. We also conclude that the system behaves asymptotically, $\phi \sim \xi \approx 2 \mathrm{H}_{0} t$; consequently, we can find the perturbed solutions when $t \rightarrow \infty$ diverge and consequently the system becomes unstable.

\section{Stability of de Sitter via the Hartman-Grobman linearizion theorem}

The stability of the dS solution plays an essential role in an inflationary scenario if we are to have a thermalization phase. Because the nonlocal theory is supposed to be an alternative for theories for inflation we will study the stability in this context.

\subsection{Stability in vacuum}

In vacuum we observed that only when $\eta \neq 0$ we have dS solutions given by (50) and (51). Let us see whether this solution is stable or not. Suppose that $H=H_{0}$, and (50) and (51) are exact solutions for (6), (7) and (38), (39) with $f(\phi)=-\phi$. We can find the following auxiliary system:

$$
\begin{aligned}
& \ddot{\xi}+3 H \dot{\xi}+6 H^{2}=0, \\
& \ddot{\phi}+3 H \dot{\phi}+6 H^{2}=0, \\
& \frac{\dot{H}}{3 H^{2}}=2 H\left(\frac{1}{\dot{\phi}}+\frac{1}{\dot{\xi}}\right)-1 .
\end{aligned}
$$

Note that Eq. $\dot{\phi}-\dot{\xi}=\frac{\eta_{0}}{a(t)^{3}}$ is obtained using subtraction of two KG eqs for $\phi, \xi$. To study stability the first step is to make system given in (63)-(65) dimensionless, using the new time parameter $N$ and the new derivative ${ }^{\prime}=\frac{d}{d N}$ we have

$$
\begin{aligned}
\varphi^{\prime} & =-6\left(2+\frac{\phi}{\alpha}\right), \\
\alpha^{\prime} & =-6\left(2+\frac{\alpha}{\varphi}\right), \\
H^{\prime} & =-3 H\left(1-\frac{2}{\varphi}-\frac{2}{\alpha}\right),
\end{aligned}
$$

where $\varphi \equiv \phi^{\prime}, \alpha \equiv \xi^{\prime}$. The critical point is located at $A=$ ( $H=H_{0}, \alpha=0, \varphi=0$ ). We linearize the system under the perturbation functions $H=H_{0}+\delta H, \varphi=\delta \varphi, \alpha=\delta \alpha$. The corresponding matrix has an eigenvalue $\lambda_{1}=0$, and it shows that the dS solution is an unstable point.

\subsection{Stability in matter mixture}

The FLRW equations are given as follows for the case with matter density and when $\phi \neq \xi$ :

$$
\begin{aligned}
& \frac{2 \dot{H}}{3 H^{2}}=\frac{-\dot{\phi} \dot{\xi}+2 H(\dot{\phi}+\dot{\xi})-\kappa \rho_{m}^{0} a(t)^{-3}}{\frac{1}{2} \dot{\phi} \dot{\xi}+\kappa \rho_{m}^{0} a(t)^{-3}}, \\
& \ddot{\xi}+3 H \dot{\xi}+6 H^{2}=0, \\
& \ddot{\phi}+3 H \dot{\phi}+6 H^{2}=0 .
\end{aligned}
$$

In this case because of $a(t)$ the system becomes nonautonomous but still we can study the local stability in the vicinity of a critical point for $t \geq t_{s}$. Using the time coordinate $N$ and by redefining $\varphi \equiv \phi^{\prime}, \alpha \equiv \xi^{\prime}$ we have the following system of differential equations:

$$
\begin{aligned}
& \frac{2}{3} \frac{H^{\prime}}{H}=\frac{H^{2}(2(\varphi+\alpha)-\varphi \alpha)-\kappa \rho_{m}^{0} a^{-3}}{\frac{1}{2} H^{2} \varphi \alpha+\kappa \rho_{m}^{0} a^{-3}} \\
& \varphi^{\prime}=\frac{-3\left(4 H^{2} a^{3} \alpha \varphi+2 H^{2} a^{3} \varphi^{2}+\kappa \varphi \rho_{m}^{0}+4 \kappa \rho_{m}^{0}\right)}{H^{2} a^{3} \alpha \varphi+2 \kappa \rho_{m}^{0}} \\
& \alpha^{\prime}=\frac{-3\left(2 H^{2} a^{3} \alpha^{2}+4 H^{2} a^{3} \alpha \varphi+\alpha \kappa \rho_{m}^{0}+4 \kappa \rho_{m}^{0}\right)}{H^{2} a^{3} \alpha \varphi+2 \kappa \rho_{m}^{0}} ;
\end{aligned}
$$

here $\varphi \equiv \phi^{\prime}, \alpha \equiv \xi^{\prime}$.

The corresponding linearized system near the unique physically accepted critical point $\{H=0, \alpha=-4, \phi=$ $-4\}$, called a proper node, or a star point (actually it defines an Einstein static solution) has a triplet proper node degenerate eigenvalue $\lambda=-\frac{3}{2}$. The critical point is asymptotically stable, and it shows that the system is asymptotically stable.

\section{Conclusions}

In this paper, we have considered the exact cosmological solutions in nonlocal $f(T)$ gravity, which can be regarded as an extension of nonlocal general relativity to the Weitzenböck spacetime. We have explored the autonomous system of the first order dynamical equations by deriving effective forms of the cosmological field equations in a homogeneous and isotropic cosmological background to describe the whole evolution history of the Universe. Furthermore, we have introduced a specific form of the interaction between matter, DE, radiation and a scalar field and analyzed the local stability in the dynamical systems, which is the so-called "stable/unstable manifold". It has been found that the system has a stable (unstable) attractor solutions. In addition, we have investigated the exact solutions of the cosmological equations in the case of de Sitter spacetime. We have demonstrated results as to whether the de Sitter solutions can exist or not in this scenario by examining the role of an auxil- 
iary function called the "gauge" function, $\eta$, in the formation of such cosmological solutions. Moreover, we have studied the stability problem of the de Sitter solutions both in vacuum and non-vacuum spacetimes. It has been shown that, for nonlocal $f(T)$ gravity, we can obtain the stable de Sitter solutions even in vacuum spacetime.

Acknowledgements We thank the anonymous referee for comments and thorough criticism on our manuscript. This work was partially supported by the JSPS KAKENHI Grant number JP 25800136 and Competitive Research Funds for Fukushima University Faculty (17RI017 and 18RI009) (K.B.).

Open Access This article is distributed under the terms of the Creative Commons Attribution 4.0 International License (http://creativecomm ons.org/licenses/by/4.0/), which permits unrestricted use, distribution, and reproduction in any medium, provided you give appropriate credit to the original author(s) and the source, provide a link to the Creative Commons license, and indicate if changes were made. Funded by SCOAP ${ }^{3}$.

\section{References}

1. A.A. Starobinsky, Phys. Lett. B 91, 99 (1980)

2. K. Sato, Mon. Not. R. Astron. Soc. 195, 467 (1981)

3. A.H. Guth, Phys. Rev. D 23, 347 (1981)

4. A.D. Linde, Phys. Lett. B 108, 389 (1982)

5. A. Albrecht, P.J. Steinhardt, Phys. Rev. Lett. 48, 1220 (1982)

6. S. Perlmutter et al. [Supernova Cosmology Project Collaboration], Astrophys. J. 517, 565 (1999). arXiv:astro-ph/9812133

7. A.G. Riess [Supernova Search Team Collaboration], Astron. J. 116, 1009 (1998). arXiv:astro-ph/9805201

8. P.A.R. Ade et al. [Planck Collaboration], Astron. Astrophys. 594, A13 (2016). arXiv:1502.01589 [astro-ph.CO]

9. P.A.R. Ade et al. [Planck Collaboration], Astron. Astrophys. 594, A20 (2016). arXiv:1502.02114 [astro-ph.CO]

10. P.A.R. Ade et al. [BICEP2 Collaboration], Phys. Rev. Lett. 112, 241101 (2014). arXiv:1403.3985 [astro-ph.CO]

11. P.A.R. Ade et al. [BICEP2 and Planck Collaborations], Phys. Rev. Lett. 114, 101301 (2015). arXiv:1502.00612 [astro-ph.CO]

12. P.A.R. Ade et al. [BICEP2 and Keck Array Collaborations], Phys. Rev. Lett. 116, 031302 (2016). arXiv:1510.09217 [astro-ph.CO]

13. E. Komatsu et al. [WMAP Collaboration], Astrophys. J. Suppl. 192, 18 (2011). arXiv:1001.4538 [astro-ph.CO]

14. G. Hinshaw et al. [WMAP Collaboration], Astrophys. J. Suppl. 208, 19 (2013). arXiv:1212.5226 [astro-ph.CO]

15. M. Tegmark et al. [SDSS Collaboration], Phys. Rev. D 69, 103501 (2004). arXiv:astro-ph/0310723

16. U. Seljak et al. [SDSS Collaboration], Phys. Rev. D 71, 103515 (2005). arXiv:astro-ph/0407372

17. D.J. Eisenstein et al. [SDSS Collaboration], Astrophys. J. 633, 560 (2005). arXiv:astro-ph/0501171

18. B. Jain, A. Taylor, Phys. Rev. Lett. 91, 141302 (2003). arXiv:astro-ph/0306046

19. S. Nojiri, S.D. Odintsov, Phys. Rep. 505, 59 (2011). arXiv:1011.0544 [gr-qc]

20. S. Nojiri, S.D. Odintsov, eConf C. 060206106 (2006) [Int. J. Geom. Meth. Mod. Phys. 4, 115 (2007)]. arXiv:hep-th/0601213

21. K. Bamba, S. Capozziello, S. Nojiri, S.D. Odintsov, Astrophys. Space Sci. 342, 155 (2012). arXiv:1205.3421 [gr-qc]

22. K. Bamba, S.D. Odintsov, Symmetry 7(1), 220 (2015). arXiv:1503.00442 [hep-th]
23. F.W. Hehl, P. Von Der Heyde, G.D. Kerlick, J.M. Nester, Rev. Mod. Phys. 48, 393 (1976)

24. K. Hayashi, T. Shirafuji, Phys. Rev. D 19, 3524 (1979). [Addendum-ibid. D 24, 3312 (1982)]

25. E.E. Flanagan, E. Rosenthal, Phys. Rev. D 75, 124016 (2007). arXiv:0704.1447 [gr-qc]

26. J. Garecki, arXiv:1010.2654 [gr-qc]

27. Y.F. Cai, S. Capozziello, M. De Laurentis, E.N. Saridakis, Rep. Prog. Phys. 79, 106901 (2016). arXiv:1511.07586 [gr-qc]

28. H.A. Buchdahl, Mon. Not. R. Astron. Soc. 150, 1 (1970)

29. S. Capozziello, S. Carloni, A. Troisi, Recent Res. Dev. Astron. Astrophys. 1, 625 (2003). arXiv:astro-ph/0303041

30. S. Nojiri, S.D. Odintsov, Phys. Rev. D 68, 123512 (2003). arXiv:hep-th/0307288

31. S.M. Carroll, V. Duvvuri, M. Trodden, M.S. Turner, Phys. Rev. D 70, 043528 (2004). arXiv:astro-ph/0306438

32. R. Ferraro, F. Fiorini, Phys. Rev. D 75, 084031 (2007). arXiv:gr-qc/0610067

33. R. Ferraro, F. Fiorini, Phys. Rev. D 78, 124019 (2008). arXiv:0812.1981 [gr-qc]

34. K. Bamba, S. Nojiri, S.D. Odintsov, Phys. Lett. B 731, 257 (2014). arXiv: 1401.7378 [gr-qc]

35. G.R. Bengochea, R. Ferraro, Phys. Rev. D 79, 124019 (2009). arXiv:0812.1205 [astro-ph]

36. E.V. Linder, Phys. Rev. D 81, 127301 (2010) [Erratum-ibid. D 82, 109902 (2010)]. arXiv:1005.3039 [astro-ph.CO]

37. K. Bamba, C.Q. Geng, C.C. Lee, L.W. Luo, JCAP 1101, 021 (2011). arXiv:1011.0508 [astro-ph.CO]

38. K. Bamba, C.Q. Geng, C.C. Lee, arXiv:1008.4036 [astro-ph.CO]

39. P. Wu, H.W. Yu, Phys. Lett. B 693, 415 (2010). arXiv:1006.0674 [gr-qc]

40. P. Wu, H. Yu, Phys. Lett. B 692, 176 (2010). arXiv:1007.2348 [astro-ph.CO]

41. S.H. Chen, J.B. Dent, S. Dutta, E.N. Saridakis, Phys. Rev. D 83, 023508 (2011). arXiv:1008.1250 [astro-ph.CO]

42. G.R. Bengochea, Phys. Lett. B 695, 405 (2011). arXiv: 1008.3188 [astro-ph.CO]

43. C.Q. Geng, C.C. Lee, E.N. Saridakis, Y.P. Wu, Phys. Lett. B 704, 384 (2011). arXiv:1109.1092 [hep-th]

44. K. Bamba, C.Q. Geng, JCAP 1111, 008 (2011). arXiv:1109.1694 [gr-qc]

45. R. Ferraro, F. Fiorini, Phys. Rev. D 84, 083518 (2011). arXiv: 1109.4209 [gr-qc]

46. H. Wei, Phys. Lett. B 712, 430 (2012). arXiv:1109.6107 [gr-qc]

47. C.Q. Geng, C.C. Lee, E.N. Saridakis, JCAP 1201, 002 (2012). arXiv: 1110.0913 [astro-ph.CO]

48. P.A. Gonzalez, E.N. Saridakis, Y. Vasquez, JHEP 1207, 053 (2012). arXiv:1110.4024 [gr-qc]

49. K. Bamba, R. Myrzakulov, S. Nojiri, S.D. Odintsov, Phys. Rev. D 85, 104036 (2012). arXiv:1202.4057 [gr-qc]

50. M. Jamil, D. Momeni, R. Myrzakulov, Eur. Phys. J. C 72, 1959 (2012). arXiv:1202.4926 [physics.gen-ph]

51. M. Jamil, D. Momeni, R. Myrzakulov, Eur. Phys. J. C 72, 2075 (2012). arXiv:1208.0025 [gr-qc]

52. M. Jamil, D. Momeni, R. Myrzakulov, Eur. Phys. J. C 72, 2122 (2012). arXiv:1209.1298 [gr-qc]

53. M. Jamil, D. Momeni, R. Myrzakulov, Eur. Phys. J. C 72, 2137 (2012). arXiv:1210.0001 [physics.gen-ph]

54. K. Izumi, Y.C. Ong, JCAP 1306, 029 (2013). arXiv:1212.5774 [gr-qc]

55. M. Jamil, D. Momeni, R. Myrzakulov, Eur. Phys. J. C 73, 2267 (2013). arXiv:1212.6017 [gr-qc]

56. K. Bamba, S.D. Odintsov, E.N. Saridakis, Mod. Phys. Lett. A 32, 1750114 (2017). arXiv:1605.02461 [gr-qc]

57. B. Li, T.P. Sotiriou, J.D. Barrow, Phys. Rev. D 83, 064035 (2011). arXiv:1010.1041 [gr-qc] 
58. T.P. Sotiriou, B. Li, J.D. Barrow, Phys. Rev. D 83, 104030 (2011). arXiv: 1012.4039 [gr-qc]

59. R. Ferraro, F. Fiorini, Phys. Lett. B 702, 75 (2011). arXiv: 1103.0824 [gr-qc]

60. M. Li, R.X. Miao, Y.G. Miao, JHEP 1107, 108 (2011). arXiv:1105.5934 [hep-th]

61. Y.C. Ong, K. Izumi, J.M. Nester, P. Chen, Phys. Rev. D 88, 024019 (2013). arXiv:1303.0993 [gr-qc]

62. K. Bamba, S.D. Odintsov, D. Sáez-Gómez, Phys. Rev. D 88, 084042 (2013). arXiv:1308.5789 [gr-qc]

63. K. Bamba, S. Capozziello, M. De Laurentis, S. Nojiri, D. SáezGómez, Phys. Lett. B 727, 194 (2013). arXiv:1309.2698 [gr-qc]

64. K. Izumi, J.A. Gu, Y.C. Ong, Phys. Rev. D 89, 084025 (2014). arXiv:1309.6461 [gr-qc]

65. P. Chen, K. Izumi, J.M. Nester, Y.C. Ong, Phys. Rev. D 91, 064003 (2015). arXiv:1412.8383 [gr-qc]

66. S. Bahamonde, C.G. Böhmer, M. Wright, Phys. Rev. D 92, 104042 (2015). arXiv:1508.05120 [gr-qc]

67. S. Deser, R.P. Woodard, Phys. Rev. Lett. 99, 111301 (2007). arXiv:0706.2151 [astro-ph]

68. S. Nojiri, S.D. Odintsov, Phys. Lett. B 659, 821 (2008). arXiv:0708.0924 [hep-th]

69. N. Arkani-Hamed, S. Dimopoulos, G. Dvali, G. Gabadadze, arXiv:hep-th/0209227

70. S. Nojiri, S.D. Odintsov, M. Sasaki, Y.L. Zhang, Phys. Lett. B 696, 278 (2011). arXiv:1010.5375 [gr-qc]

71. K. Bamba, S. Nojiri, S.D. Odintsov, M. Sasaki, Gen. Relativ. Gravit. 44, 1321 (2012). arXiv:1104.2692 [hep-th]

72. Y.L. Zhang, M. Sasaki, Int. J. Mod. Phys. D 21, 1250006 (2012). arXiv:1108.2112 [gr-qc]

73. L. Parker, D.J. Toms, Phys. Rev. D 32, 1409 (1985)

74. T. Banks, Nucl. Phys. B 309, 493 (1988)

75. C. Wetterich, Gen. Relativ. Gravit. 30, 159 (1998). arXiv:gr-qc/9704052

76. A.O. Barvinsky, Phys. Lett. B 572, 109 (2003). arXiv:hep-th/0304229

77. J. Khoury, Phys. Rev. D 76, 123513 (2007). arXiv:hep-th/0612052

78. L. Joukovskaya, Phys. Rev. D 76, 105007 (2007). arXiv:0707.1545 [hep-th]

79. G. Calcagni, M. Montobbio, G. Nardelli, Phys. Lett. B 662, 285 (2008). arXiv:0712.2237 [hep-th]

80. S. Jhingan, S. Nojiri, S.D. Odintsov, M. Sami, I. Thongkool, S. Zerbini, Phys. Lett. B 663, 424 (2008). arXiv:0803.2613 [hep-th]

81. T. Koivisto, Phys. Rev. D 77, 123513 (2008). arXiv:0803.3399 [gr-qc]

82. N.A. Koshelev, Gravit. Cosmol. 15, 220 (2009). arXiv:0809.4927 [gr-qc]

83. M.G. Romania, N.C. Tsamis, R.P. Woodard, Lect. Notes Phys. 863, 375 (2013). arXiv:1204.6558 [gr-qc]

84. A.O. Barvinsky, Y.V. Gusev, Phys. Part. Nucl. 44, 213 (2013). arXiv:1209.3062 [hep-th]

85. S. Deser, R.P. Woodard, JCAP 1311, 036 (2013). arXiv: 1307.6639 [astro-ph.CO]

86. M.M. Ivanov, A.V. Toporensky, Gravit. Cosmol. 18, 43 (2012). arXiv: 1106.5179 [gr-qc]

87. R.D. Boko, M.J.S. Houndjo, J. Tossa, Int. J. Mod. Phys. D 25, 1650098 (2016). arXiv:1605.03404 [gr-qc]

88. S.D. Odintsov, V.K. Oikonomou, P.V. Tretyakov, Phys. Rev. D 96, 044022 (2017). arXiv:1707.08661 [gr-qc]

89. S.D. Odintsov, V.K. Oikonomou, Phys. Rev. D 93, 023517 (2016). arXiv: 1511.04559 [gr-qc]

90. C.G. Boehmer, T. Harko, S.V. Sabau, Adv. Theor. Math. Phys. 16, 1145 (2012). arXiv:1010.5464 [math-ph]

91. N. Goheer, J.A. Leach, P.K.S. Dunsby, Class. Quantum Gravity 24, 5689 (2007). arXiv:0710.0814 [gr-qc]
92. G. Leon, E.N. Saridakis, JCAP 1504, 031 (2015). arXiv: 1501.00488 [gr-qc]

93. G. Leon, E.N. Saridakis, Class. Quantum Gravity 28, 065008 (2011). arXiv:1007.3956 [gr-qc]

94. J.C.C. de Souza, V. Faraoni, Class. Quantum Gravity 24, 3637 (2007). arXiv:0706.1223 [gr-qc]

95. A. Giacomini, S. Jamal, G. Leon, A. Paliathanasis, J. Saavedra, Phys. Rev. D 95, 124060 (2017). arXiv:1703.05860 [gr-qc]

96. G. Kofinas, G. Leon, E.N. Saridakis, Class. Quantum Gravity 31, 175011 (2014). arXiv:1404.7100 [gr-qc]

97. G. Leon, E.N. Saridakis, JCAP 1303, 025 (2013). arXiv:1211.3088 [astro-ph.CO]

98. T. Gonzalez, G. Leon, I. Quiros, Class. Quantum Gravity 23, 3165 (2006). arXiv:astro-ph/0702227

99. A. Alho, S. Carloni, C. Uggla, JCAP 1608, 064 (2016). arXiv:1607.05715 [gr-qc]

100. D. Moller, V.C. de Andrade, C. Maia, M.J. Rebouas, A.F.F. Teixeira, Eur. Phys. J. C 75, 13 (2015). arXiv:1405.0768 [astro-ph.CO]

101. S. Rippl, H. van Elst, R.K. Tavakol, D. Taylor, Gen. Relativ. Gravit. 28, 193 (1996). arXiv:gr-qc/9511010

102. M. Maggiore, Fundam. Theor. Phys. 187, 221 (2017). arXiv:1606.08784 [hep-th]

103. G. Otalora, E.N. Saridakis, Phys. Rev. D 94(8), 084021 (2016). $\quad$ https://doi.org/10.1103/PhysRevD.94.084021. arXiv: 1605.04599 [gr-qc]

104. S. Bahamonde, S. Capozziello, M. Faizal, R.C. Nunes, Eur. Phys. J. C 77, 628 (2017). arXiv:1709.02692 [gr-qc]

105. P. Channuie, D. Momeni, Nucl. Phys. B 935, 256 (2018). https:// doi.org/10.1016/j.nuclphysb.2018.08.016. arXiv:1712.07927 [gr-qc]

106. K. Hayashi, T. Shirafuji, Phys. Rev. D 19, 3524 (1979). Addendum: [Phys. Rev. D 24, 3312 (1982)]

107. S. Bahamonde, S. Capozziello, K.F. Dialektopoulos, Eur. Phys. J. C 77, 722 (2017). arXiv:1708.06310 [gr-qc]

108. A. Awad, W. El Hanafy, G.G.L. Nashed, E.N. Saridakis, JCAP 1802(02), 052 (2018). arXiv:1710.10194 [gr-qc]

109. M. Jamil, K. Yesmakhanova, D. Momeni, R. Myrzakulov, Central Eur. J. Phys. 10, 1065 (2012). arXiv:1207.2735 [gr-qc]

110. Yu. Pan, L. Li, S. Cao, P.-N. Pan, Y. Zhang, Z.-X. Hu, Testing the interaction between dark energy and dark matter with $H(z)$ data. Chin. Astron. Astrophys. 40(2), 176-185 (2016). https://doi.org/ 10.1016/j.chinastron.2016.05.007. (ISSN 0275-1062)

111. N. Cruz, S. Lepe, F. Pena, Phys. Lett. B 663, 338 (2008). arXiv:0804.3777 [hep-ph]

112. N. Cruz, S. Lepe, F. Pena, Phys. Lett. B 699, 135 (2011)

113. M. Jamil, E.N. Saridakis, M.R. Setare, Phys. Rev. D 81, 023007 (2010). arXiv:0910.0822 [hep-th]

114. M. Jamil, D. Momeni, M.A. Rashid, Eur. Phys. J. C 71, 1711 (2011). arXiv:1107.1558 [physics.gen-ph]

115. S. Chen, J. Jing, Class. Quantum Gravity 26, 155006 (2009). arXiv:0903.0120 [gr-qc]

116. J.H. He, B. Wang, E. Abdalla, Phys. Rev. D 83, 063515 (2011). arXiv:1012.3904 [astro-ph.CO]

117. A.A. Coley, Dynamical Systems and Cosmology, vol. 291 (Kluwer, Dordrecht, 2003). https://doi.org/10.1007/ 978-94-017-0327-7

118. G. Leon, C.R. Fadragas, Cosmological Dynamical Systems (LAP Lambert Academic Publishing, Saarbrücken, 2012)

119. C.G. Boehmer, N. Chan, Dynamical systems in cosmology. LTCC Advanced Mathematics Series: Dynamical and Complex Systems, 5, 121-156 (2017), https://doi.org/10.1142/ 9781786341044_0004. arXiv:1409.5585 [gr-qc]

120. J. Dutta, W. Khyllep, E.N. Saridakis, N. Tamanini, S. Vagnozzi, JCAP 1802, 041 (2018). arXiv: 1711.07290 [gr-qc]

121. M. Betoule et al. [SDSS Collaboration], Astron. Astrophys. 568, A22 (2014). arXiv:1401.4064 [astro-ph.CO] 
122. F. Beutler, C. Blake, M. Colless, D.H. Jones, L. Staveley-Smith, L. Campbell, Q. Parker, W. Saunders, F. Watson, Mon. Not. R. Astron. Soc. 416, 3017 (2011). arXiv:1106.3366 [astro-ph.CO]

123. A.J. Ross, L. Samushia, C. Howlett, W.J. Percival, A. Burden, M. Manera, Mon. Not. R. Astron. Soc. 449, 835 (2015). arXiv:1409.3242 [astro-ph.CO]

124. L. Anderson et al. [BOSS Collaboration], Mon. Not. R. Astron. Soc. 441, 24 (2014). arXiv:1312.4877 [astro-ph.CO]

125. A. Font-Ribera et al. [BOSS Collaboration], JCAP 1405, 027 (2014). arXiv:1311.1767 [astro-ph.CO]
126. R.C. Nunes, S. Pan, E.N. Saridakis, E.M.C. Abreu, JCAP 1701, 005 (2017). arXiv:1610.07518 [astro-ph.CO]

127. M. Moresco, R. Jimenez, L. Verde, A. Cimatti, L. Pozzetti, C. Maraston, D. Thomas, JCAP 1612, 039 (2016). arXiv:1604.00183 [astro-ph.CO]

128. S. Capozziello, V.F. Cardone, H. Farajollahi, A. Ravanpak, Phys. Rev. D 84, 043527 (2011). arXiv:1108.2789 [astro-ph.CO]

129. P. Wu, H. Yu, Phys. Lett. B 703, 223 (2011). arXiv:1108.5908 [gr-qc] 